\title{
DESIGN AND DEVELOPMENT OF A MICROCONTROLLER BASED PORTABLE ECG MONITOR
}

\author{
Nahian Rahman', A K M Bodiuzzaman, A Raihan Abir, K Siddique-e Rabbani \\ Department of Biomedical Physics \& Technology, University of Dhaka, Dhaka, Bangladesh \\ ${ }^{1}$ Dept of Mechanical Engineering, BUET, Dhaka, Bangladesh \\ email: nahiian@yahoo.com,nipun1207@gmail.com,raihan1079@gmail.com,rabbani@univdhaka.edu
}

\begin{abstract}
A portable battery powered miniature ECG monitor with built in graphic display has been developed at low cost which can be used in rural areas, for on site patient service. It involves an analogue electronics circuit and a programmable digital circuit in the form of a Microcontroller unit (MCU). The MCU sends necessary data to an LCD graphic display screen having 128 x 64 pixels and a built-in controller. The data acquisition, processing and plotting of graphs on the screen in real time are performed through a software developed using ' $\mathrm{C}$ ' language. The hardware was mounted in a compact box with integrated battery. Field trial and further improvement will be needed before it can be given out to users. A little modification will make it suitable for continuous ambulatory monitoring, with data stored in an SDRAM, which can later be read into a PC and analysed by a doctor.
\end{abstract}

Keywords: Portable ECG, Rural healthcare,

\section{INTRODUCTION}

Electrocardiogram (ECG) indicates the electrical activity of the heart of a human body. The heart has a system of localized electrical oscillators that generate pulses to activate the heart muscles through specialized connective tissues, all under the regulation of the brain. Since the pumping action of the heart depends on these electrical activities, obtaining ECG is very important from a clinician's point of view. It gives the heart-beats, the size and position of the chambers, presence of any damage to the heart and the effects of drugs or devices used to regulate the heart. Many ECG equipment are available in the market but the prices are very high, and are beyond the affordability of rural health centres in low income countries like Bangladesh. The technological and economic disparity created between countries of the world, and commercialization of such equipment by manufacturers in only rich countries have caused an unwelcome situation whereby a majority of global population living in the low income countries are yet to get the benefits of such modern methods of healthcare. The ECG equipment was invented more than a hundred years back, but still a large majority of the global population is yet to get the benefit of this technology. This is an undesirable situation and a remedy is only possible if such healthcare equipment are developed indigenously in each country [1,2]. This was the motivation to develop an ECG monitor indigenously by developing our own technological expertise. Our extended group has also developed a PC based ECG equipment [3] which may be used in rural health centres, and may contribute to indigenous efforts to develop telemedicine. The present work was taken up in parallel to develop a miniature portable ECG monitor, operated by a battery, which may also be used in a rural health centre, but the main benefit is that mobile village doctors may carry this equipment in their bags and provide service to people at their own homestead.

\section{METHODS}

ECG is a slow waveform, and typically an ECG equipment should display real time data recorded over a few seconds on a monitor or screen. This needs the storage of data in an electronic memory and its simultaneous display in an electronic display device. In the present work an LCD monochrome display was used for this purpose and a controller chip integrated to it stored the collected data for the necessary display period. A microcontroller unit (MCU) with a built-in analogue to digital converter (ADC) was 
used to convert the analogue ECG signal to a binary digital form. The same MCU was used to configure the display controller, establish the co-ordinates of display, and to send the converted digital data for real

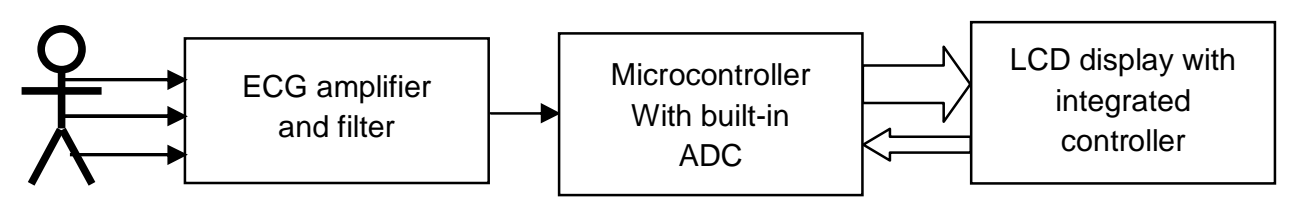

Fig. 1: Block Diagram of the portable ECG system

time display of the ECG signal. Necessary hardware and software were designed and developed in the present work. In conventional ECG equipment powered by mains ac, electrical isolation of the patient side is necessary to avoid microshock hazards. However, in this design the whole equipment is battery powered, and therefore, electrical isolation is not necessary, which simplifies the analogue circuitry. Fig.1 shows a block diagram. The hardware and software parts are described separately below.

\subsection{Hardware part}

The ECG waveforms collected from a subject is of analogue type while acquisition, storage and display requires the data to be converted and handled using digital techniques. Therefore, the hardware can be separated into two parts - analogue and digital, and these are described separately below.

\subsubsection{Analogue part}

The developed circuitry is shown in Fig.2. AMP-02 is an instrumentation amplifier IC which has a differential input and a single ended output. That is it has two input ports and 3 leads, including a common, which are taken out to the patient. Its gain is controllable using $\mathrm{R}_{\mathrm{G}}$. A low gain of about 10 has been chosen so that the output does not become saturated because of contact potential developed at the electrodes. AMP-02 has high input impedance and a Common Mode Rejection Ratio (CMRR) of 115dB at such gains, which is adequate for the ECG system. The output is filtered to remove low and high frequency noise, and its de level is shifted to match that of the ADC input of the microcontroller unit (MCU). Since ECG signal may have both positive and negative parts, while the MCU works with single ended power, this dc shift is necessary.

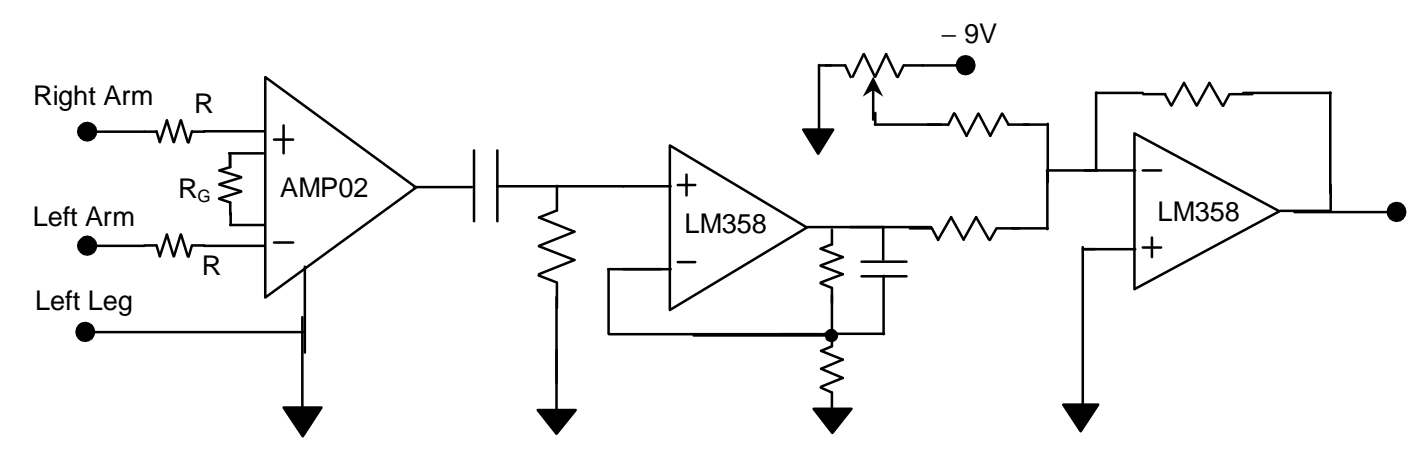

Fig. 2: Analogue part of the portable ECG system 
The ECG amplifier is powered by two 9 volt batteries for the positive and the negative supplies. The MCU \& display require +5 volt which is obtained from the $+9 \mathrm{~V}$ supply through a voltage regulator IC. The analogue output that is provided for the ADC inside the MCU ranges between $0 \mathrm{~V}$ and $3 \mathrm{~V}$.

\subsubsection{Digital part}

This is built around an Atmega32 microcontroller unit (MCU), having a built-in 10-bit successive approximation Analogue to Digital Converter (ADC) and multiplexed 8-channel analog inputs. The conversion time is about $13 \mu \mathrm{Sec}$. An internal RC Oscillator was used as a clock source for the MCU having a clock frequency of $1 \mathrm{MHz}$. The analogue input range selected was $0 \mathrm{~V}$ to $+\mathrm{V}_{\mathrm{CC}}$, where $+\mathrm{V}_{\mathrm{CC}}$ is the supply voltage, which, in this case was $+5 \mathrm{~V}$. The ADC has two modes of operation, Free Running or Single Conversion Mode, the latter was chosen in this work.

The MCY has four ports, A, B, C and D for various input/output (I/O) operations, as programmed by the software. There are 8 multiplexed analogue inputs through Port A which connects these to a built in ADC. The use of the other ports are described below, and shown in the pin diagram of the IC in Fig. 3.

The Graphical Liquid Crystal Display (GLCD) chosen is 'Topway LM6063' and it is driven by a integrated controller unit 'KS0713' and operates in 8-bit mode. The GLCD has a monochrome screen with a resolution of $128 \times 64$, and with white dots displayed on a blue background. The GLCD is also an intelligent device together with the built-in controller, and it realizes the significance of the data and executes the command, according to the status available through the control pins. Through the specified controller the display is built up as 8 horizontal lines, one below the other; each line built up as 8 dots arranged sequentially in 128 consecutive horizontal positions. Although bitmap displays would have been better, their unavailability in the local market made us to choose the above. Both the Microcontroller and the GLCD were chosen based on local availability and cost.

The output signal from the ECG amplifier was fed to the MCU through PA0 pin, an analogue input port $(\mathrm{ADC} 0)$ of Port A. Outputs of the MCU are obtained from Port C \& Port D. The MCU was connected to the data pins \& control pins of the integral controller of the GLCD through 13 connections. 8 data pins of Port C (PC0 to PC7) and 5 control pins of Port D (PD3 to PD7) were connected to the GLCD controller. The signals provided at the Control pins by the MCU suggest the GLCD controller about the data $\&$ what to do with the data.

The developed system has five buttons to control the graphical display. Two buttons provide shifting of the display upward \&

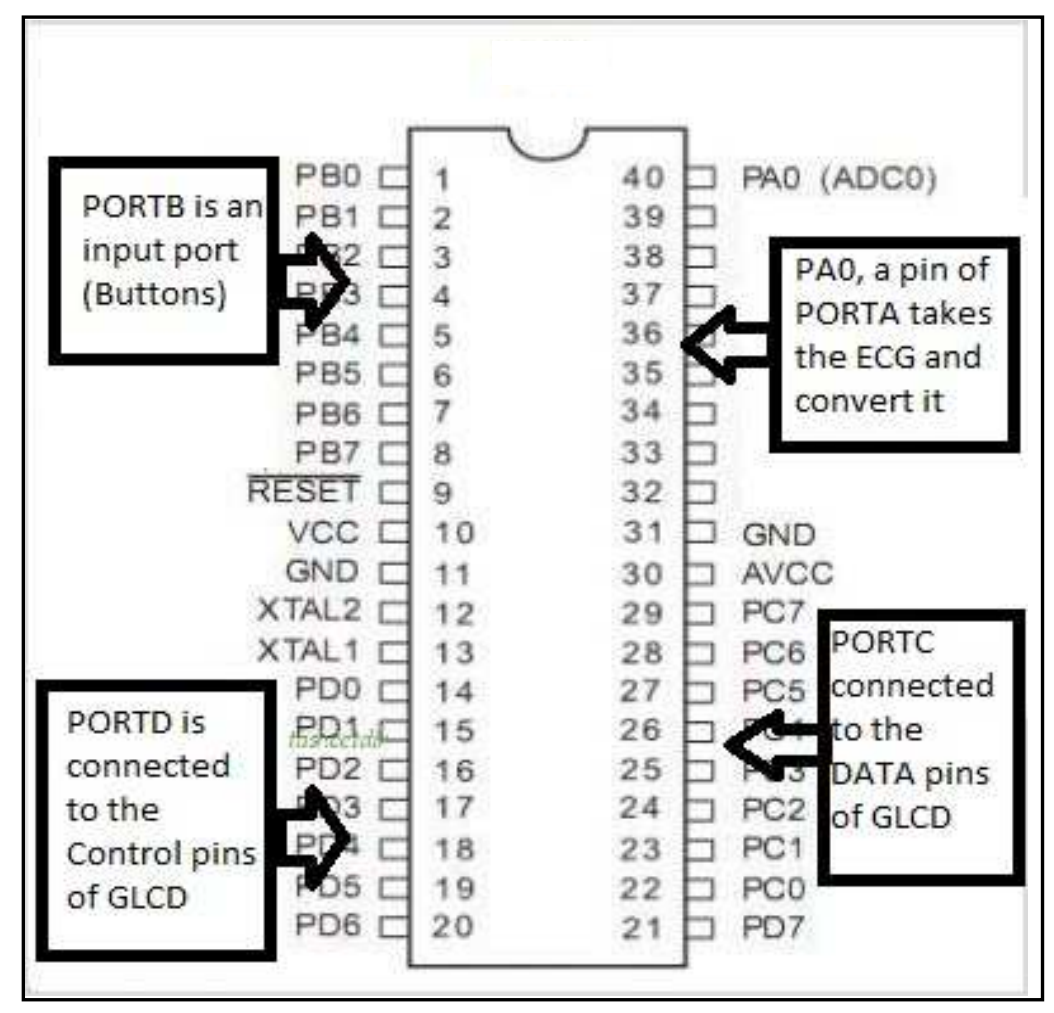

Fig.3: Pin diagram of Atmega32 Microcontoller unit 
downward, while another two expands or contracts the display horizontally. The latter is basically done through changing the sampling rate of data acquisition. The fifth button provides a freezing action on the display, allowing it to be observed as long as desired. The buttons are connected to Port B (PB0 to PB4) of the MCU.

\subsection{Programming the MCU}

The program written into the MCU has been separated into two parts, 'Firmware, and 'Software'. The program that controls the basic interfacing and communication between the MCU and the GLCD controller has been named the firmware.

The program that performs all other functions of acquiring analogue data samples at chosen intervals of time, sending the converted digital data to the GLCD controller, and calculating the positions on the screen where to place a dot display for the desired graphical output, has been named the software. This also controls the operation of the five buttons which allows the MCU to send the appropriate control signals to the ADC and the GLCD controller, and provides a time scale on the display using the built-in timer counter of the MCU. The software was developed in ' $\mathrm{C}$ ' language using an integrated development environment named 'AVR Studio'.

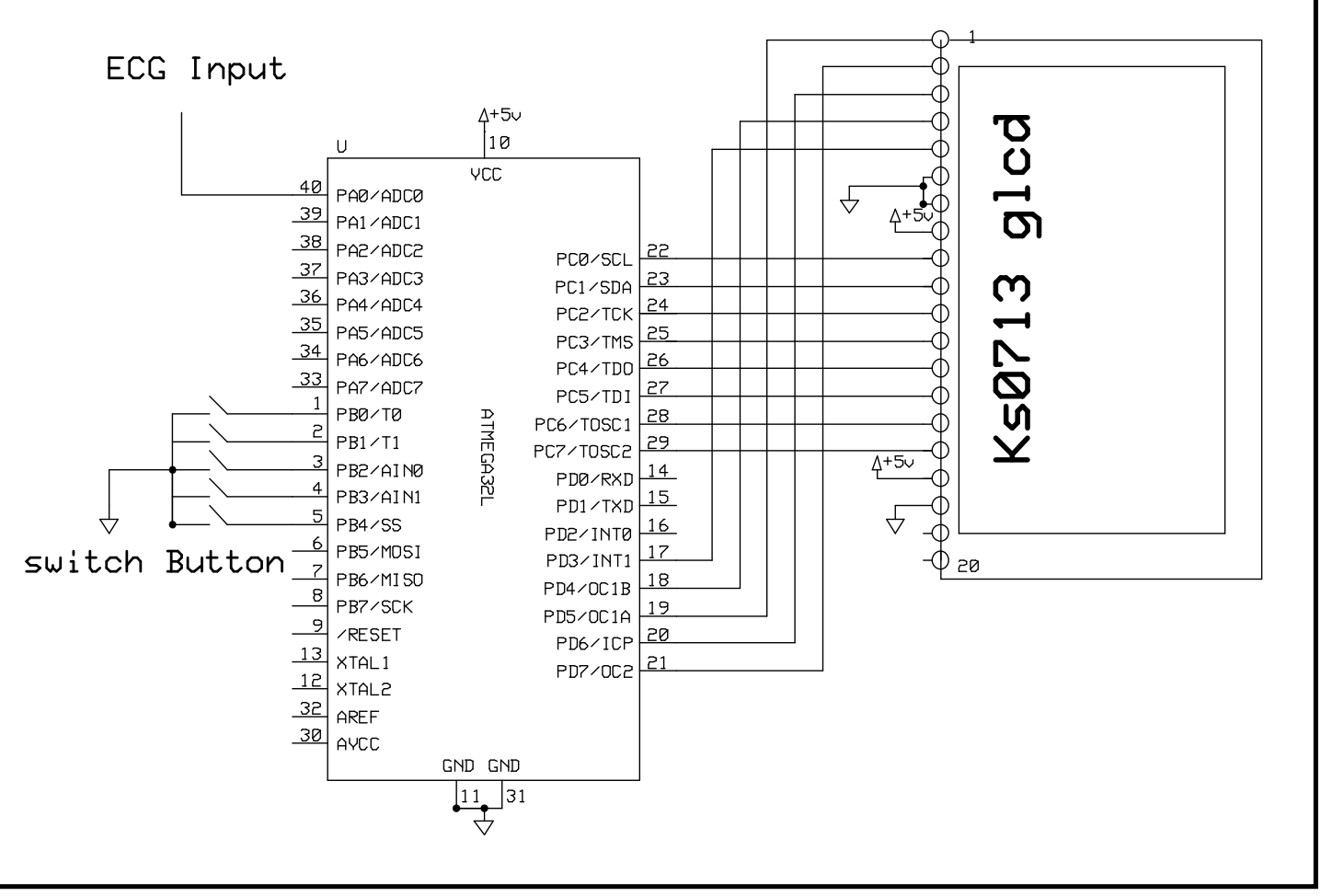

Fig.4: Interfacing of Atmega32 and GLCD Controller

To summarise, the software performs the following functions

- Input and data conversion (ADC), and output to GLCD controller

- Graphics control

- Timer Counter (needed for screen calibration) 


\subsubsection{Firmware development for GLCD controller}

As mentioned before, the screen of the Topway LM6063 has 128 x 64 pixels and its controller, Ks0713, performs the addressing of pixels in a row-column mapped way which is different from a bit mapped display commonly used for graphics. In a bit mapped display, whenever a dot is to be plotted, it is addressed directly to the co-ordinates of the desired pixel position. The GLCD controller on the other hand sends data in bytes (packet of 8 bits) which take up a column within a row of 128 pixels. That means one row of display (called a line) is made of $128 \times 8$ pixels. There are 8 of these rows arranged vertically one below the other to make up the full $128 \times 64$ pixel display. Therefore, necessary calculations have to be made to determine which of these vertical 8 pixels have to be displayed using the data byte sent. This has to be done in the software by producing a code that will firstly calculate the line number, and then the column number within each line, and then the bits within each byte of this column that will appear as displayed dots. The graphical arrangement and the addresses for each row and column are shown in Fig.5.

The firmware part was written with the guidance of Ks0713 controller's datasheet. To initialize the GLCD some sequential commands had to be maintained. For all the commands, first a given pattern was sent to the control pins. Then the data was sent to the data pins. The origin $(0,0)$ of the screen coordinates start from the top-leftmost pixel. For example, for a dot to be displayed at this position, the column address had to be set to 0 (leftmost column), the page address (giving the line no.) had to be set to 0 , while the binary data value (8-bit) had to be set to 00000001, in the sequence, $0,0,00000001$. The GLCD controller takes and performs the necessary display according to the status of control pins. Similarly to display a dot at the top-rightmost pixel, the command data had to be in the sequence, 127,0, 00000001.

\begin{tabular}{|c|c|c|c|c|}
\hline Page address & data & & LCD Display (front view) & \\
\hline \multirow{2}{*}{0} & D0 & & & \\
& $\vdots$ & & & \\
D7 & & & \\
\hline & D0 & & & \\
\hline & D7 & & & \\
\hline & D0 & & & 127 \\
\hline
\end{tabular}

Fig. 5: Address scheme of the 128 x 64 Graphical LCD screen through the controller

In order to give a horizontal time scaling, a numerical value is printed on the top-left corner of the screen display which equals the time duration that a full horizontal display takes. This timing may be changed by the user through the two external horizontal buttons described before. This essentially changes the screen display by expanding or compressing the placement of the dots horizontally rather than changing the sampling rate of the input signal, because a low sampling rate might lead to missing of vital data. 
The ADC inside the Atmega 32 produces 10 bit binary data while the GLCD takes 8 bit data. For simplicty and for faster performance, the most significant 8 bits of the converted value was taken. Leaving the two lower bits out causes errors of less than $0.3 \%$ of the original value which can be ignored.

Since the ECG is plotted in real time, several things are to be considered. First thing is the format of ADC conversion. There are two way of converting ADC, free running mode, and step mode. In free running mode, there is an interrupt which informs the main program when the conversion is complete. Use of this interrupt is attractive, but it may give rise to unequal sample intervals since all data conversions may not take exactly the same time interval. Besides, this will not allow easy change of sampling interval if desired. Furthermore, since the GLCD needs some time to plot a point, erroneous data acquisition and display may take place if this interrupt appears during the plotting period. In the step mode the sampling of input data is done on command from the main programme. Therefore, it may be performed in equal intervals of time provided adequate delay is allowed for conversion of data by the ADC and plotting of the point in the GLCD. ECG is a slow signal, and therefore the sampling intervals needed are large enough to satisfy the above condition with ease. Therefore, in the present work the step mode was used.

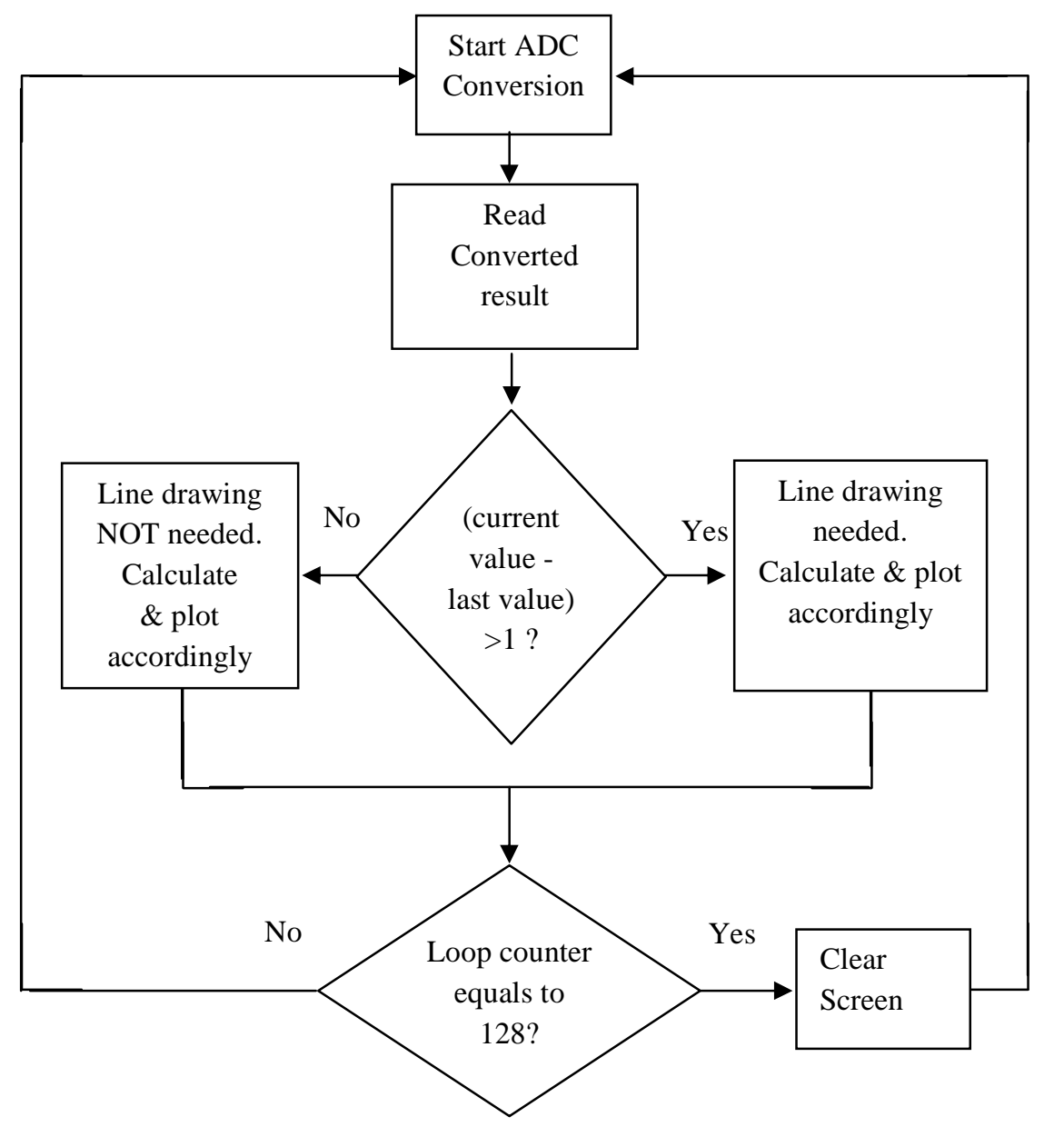

Fig.6: Program flow diagram including that of drawing of lines between two adjacent points. 


\subsubsection{Time calibration}

The time calibration of the horizontal display is shown numerically on the top-left corner of the screen, in terms of the time required to plot a whole horizontal trace, consisting of the full 128 columns. This timing was obtained from a 16 bit Timer/Counters (Timer 1) available in the MCU. It takes pulses form the internal oscillator which was driven in the present design at $1 \mathrm{MHz}$ through the internal RC configuration. At the end of plotting the full horizontal line (128 columns) the total number of pulses occurring during the line plotting since its beginning is calculated to give this time. The whole screen is then erased and the process is repeated in an endless loop. Within this loop, the program checks certain things as follows.

- Checks the five buttons (Input from user)

- Compares the input voltage with a reference, gives a value, and saves it for further comparison

- Prints a dot display at the calculated current cursor position in the GLCD

- Increases the Cursor position bar

- Erases the screen when a horizontal plotting is completed.

- Calculates total elapsed time and prints it at the top-left position of the screen

\subsubsection{Line joining program algorithm}

In an ECG there are segments with a significantly fast rise and fall time. Therefore, adjacent horizontally displayed points may have a significant vertical gap making the display of the ECG waveform rather dotty, and not acceptable to the user. Therefore, a programme was developed to 'fill in the gap' using more dots in both the vertical columns, to look like a continuous unbroken line display. Whenever the current plotting value has a difference of more than 1 compared to the last plotted value, this routine comes into play. The flow diagram, including that of the data acquisition is given in Fig.6.

\section{RESULTS AND OBSERVATIONS}

The gain of the amplifier had to be adjusted to get a reasonable amplitude of ECG in the display. An initial programme value sets the timing of the horizontal display. The horizontal scale was fine tuned using the push button controls to get a number of ECG peaks in the display. A resulting ECG display from a human subject as obtained in the prototype is shown in Fig.7.

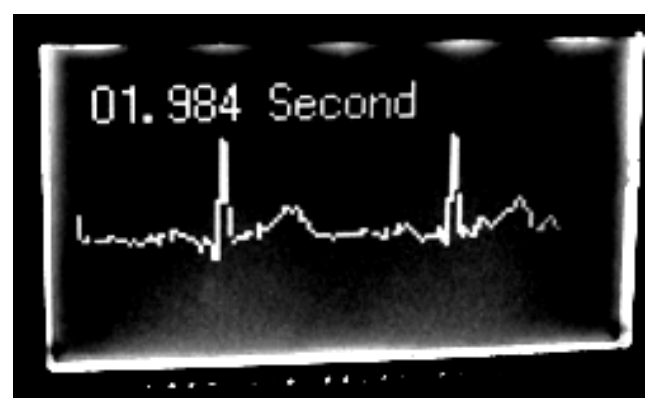

Fig.7: ECG display in the experimental prototype

\section{DISCUSSION}

The portable miniature ECG equipment was designed for rural health centres and for mobile doctors. The cost and simplicity of use were of concern too. The device has been made out of components that are available widely in the world market, and most of them are available ready stock in Bangladesh. As can 
be seen from Fig.7, The system shows satisfactory results in a prototype. The prototype has been housed in a small plastic case, including batteries, making it convenient to use. Field trial and further improvement will be needed before it can be given to users.

A right leg drive may be incorporated in the analogue part to reduce common mode noise. Some protection circuitry may be added at the input as well. The three leads currently provided are suitable for classical limb lead ECG's in the frontal plane (Lead I, II and III). However, using a switch box with suitable resistors in the front end, other configurations of ECG's may be obtained. Using Windows Hyper Terminal the data can be send to PC, curves can displayed in Microsoft Excel, Origin or other graph plotting software. More hardware buttons can be added for various purposes like start/stop recording, vertical axis magnification, etc. Further improvement can be made by putting calibration marks and scales directly along the axes of the graph. Heart beat rates may be calculated from the data and displayed on the screen.

Further signal conditioning may be performed in the MCU to reduce the noise. Besides, more facilities may be added in future, like transfer data to a PC directly, storing a large number of data in a SDRAM which can be read in a PC later. The latter may open the possibility for ambulatory monitoring over 24 hours. Printing ECG waveforms directly from the portable unit may be possible by using MCU's with more advanced features, which have printer peripherals inbuilt. The prototype may also be used as a data logger for analogue inputs of various signals. The wave forms may be stored in the built-in EEPROM, or in a flash memory, and played back for viewing again.

Therefore, the present effort may contribute immensely in bringing modern healthcare technology for the benefit of the common people in the low income countries of the world.

\section{REFERENCES}

1. Rabbani K S, Local development of Biomedical Technology - a must for the Third World, Proceedings, Regional Conference of IEEE-EMBS and 14th BMES- India, Delhi,p.1.31-1.32, 1995

2. Rabbani K S, How to deliver the benefits of modern Biomedical Engineering to the common people in the economically developing countries, Invited Lecture, 9th Medical Engineering Conference, Yamagata, Japan, October 26-27, 1995.

3. Rabbani K S, Abir A R, Bodiuzzaman A K M, Design and Development of Low Cost Personal Computer Based ECG Monitor, Bangl;adesh Journal of Medical Physics, Vol.4, January 2011 (current issue). 